\title{
„Bildung“ - ein Thema im Dissens der Disziplinen
}

\section{$Z f E$}

\author{
Heinz-Elmar Tenorth
}

„Bildung“ - was immer das schließlich ist - gehört bekanntlich zu den Themen, von denen nicht nur die Wissenschaft, sondern auch die Journaille, die Politik und der Alltag voll sind, und zwar in ganz heterogener Weise. „Bildung macht reich“, das verspricht im Sommer 2009 die Einladung zu einer Podiumsdiskussion, - und Veranstalter ist nicht etwa der Bundesverband der Deutschen Industrie, sondern der Managerkreis der sozialdemokratischen Friedrich-Ebert-Stiftung (den es also gibt). „Bildung für alle“, das sei das gegenwärtige Äquivalent für Ludwig Erhards alten Slogan vom „Wohlstand für Alle" und das sagt natürlich die CDU, ebenfalls 2009, durch die Bundeskanzlerin höchstpersönlich, wenn sie die „Bildungsgesellschaft“ verkündet (und nebenher immerhin einräumt, dass Wohlstand nicht mehr das universale Versprechen ist). Das Publikum nimmt solche Thesen offenbar dankbar auf: Die einschlägigen Seiten der großen Zeitungen heißen „Bildungswelten“, „Chancen“ und „Bildung und Karriere“. Auf die Frage nach dem wichtigsten Thema für ihre Zukunft antworten die Jugendlichen in der jüngsten Shell-Studie jedenfalls „Bildung“ und was damit ermöglicht werden soll, der universale Wunsch der guten Zukunft, das konnte man jüngst im Kabarett hören, natürlich triadisch: ein 3er-BMW, ein gut bezahlter Job und eine schöne Freundin.

Gegen solch alltägliche Visionen, kritisch zugespitzt als Tendenz zur „Ökonomisierung der Bildung“, demonstrierten dagegen im Juni 2009 in ihrem „Bildungsstreik“ mehr als 200.000 Schüler und Studierende für eine „Zweckfreie Bildung“, unter Berufung auf Wilhelm von Humboldt, im Konsens mit einer großen Zahl ihrer Professoren, im Übrigen: nicht nur aus den Humanwissenschaften - und auf keiner Demonstration oder Diskussionsveranstaltung haben sie sich mit der Aussicht auf Reichtum trösten lassen, vielmehr die Kosten der Bildung, vor allem die Studiengebühren, als Mechanismus des

Online publiziert: 19.08 .2011

(C) Die Autor(en) 2011. Dieser Artikel ist auf Springerlink.com mit Open Access verfügbar.

Vortrag am 1.6.2011 anlässlich der Verleihung der Ehrendoktorwürde durch die Fakultät für Philosophie und Erziehungswissenschaft der Ruhr-Universität Bochum.

Prof. Dr. Dr. h.c. H.-E. Tenorth $(\bowtie)$

Institut für Erziehungswissenschaften, Abteilung Historische Erziehungswissenschaft,

Humboldt Universität zu Berlin, Unter den Linden 6, 10099 Berlin, Deutschland

E-Mail: tenorth@cms.hu-berlin.de 
Ausschlusses von Bildung verurteilt. Andererseits stellen Bildungsforscher die Ausbreitung von „Bildungsarmut“ fest und sie bezeichnen damit die Tatsache, dass die Zahl der Schulabsolventen unvermindert hoch bleibt, die nicht über Bildungsabschlüsse verfügen und die auch nicht die Kompetenzen erworben haben, um erfolgreich eine berufliche Ausbildung aufzunehmen oder sich durch Bildung gegen Arbeitslosigkeit zu sichern oder selbstbestimmt an Gesellschaft und Kultur teilzunehmen, um sich auch ökonomisch selbst reproduzieren zu können. In den „Wissensgesellschaften“, in die wir eingetreten sein sollen, ist eine Existenz ohne hinreichende „Bildung“ offenbar doch ein Ausschluss gravierender Art; jedenfalls ist man von dem kollektiven „Aufstieg durch Bildung“ weit entfernt, der zu Beginn der Bildungsexpansion in den 1960er-Jahren so laut versprochen wurde. Aber eingetreten ist trotz einer starken Zunahme an Abiturienten - von etwa 5 auf nahezu $40 \%$ des Altersjahrgangs - nicht mehr Gleichheit in der Gesellschaft, sondern fortdauernde Ungleichheit, wenn auch auf einem höheren Niveau („Fahrstuhleffekt“ nennen das die Bildungssoziologen, wenn wir alle nach oben befördert werden, uns dann aber in der gleichen Hierarchie, nur eine Etage höher, wieder finden).

„Die höchste und proportionierlichste Bildung aller Kräfte zu einem Ganzen“ (Humboldt 1792/1980a, S. 64), wie Bildung in den klassischen Texten bei und seit Wilhelm von Humboldt beschworen wird, die Verbreitung des „Wahren, Schönen und Guten“ und die „Höherbildung der Menschheit“ - das kann man vielleicht noch in Festreden hören, es ist heute jedenfalls nicht der einzige Ton, in dem über Bildung gesprochen wird. Das ist auch verständlich, denn die Rede über Bildung ist schon immer vielgestaltig gewesen: Sie erörtert die Tugenden und Leistungen der Subjekte genauso wie die Strukturprinzipien von Bildungssystemen oder die Leitformeln für die Identität einer Nation. „Bildung“ ist ein deutscher Mythos, ist pädagogisches Programm, ist politische Losung, ist Ideologie des Bürgertums und zugleich doch auch ein viel genutzter zeitdiagnostischer Kritikbegriff.

Gibt es in dieser Mehrdimensionalität der Rede und der Funktion von Bildung noch Konsenszonen? Solche, die es erlauben, die öffentliche Rede zu ordnen und zu problematisieren und den Sinn der Rede von Bildung auch noch theoretisch zu verstehen? Man würde sich das von den „Bildungswissenschaften“ wünschen, von denen neuerdings die Rede ist, als Oberbegriff für eine ebenfalls sehr disparate Gruppe von Disziplinen: Unter den „Bildungswissenschaften“ findet man nicht nur die Erziehungswissenschaft oder die Pädagogische Psychologie bzw. heute schon die „Bildungspsychologie“, sogar mit eigenem disziplinären Anspruch, auch die Fachdidaktiken oder, in bildungswissenschaftlichen Hochschulen, die Bildungssoziologie und generell alle Arten empirischer Bildungsforschung, bis hin zur Bildungsökonomie oder zum Bildungsrecht; und in der wissenschaftlichen Kommunikation melden sich auch Neurowissenschaften sowie Neuropädagogik und -didaktik bei diesem Thema zu Wort. Bildung ist daneben, natürlich, weiterhin Thema in den Literatur- und Kulturwissenschaften oder in der Geschichte, nicht nur des Bildungsbürgertums. Es gibt offenbar keine klar definierten disziplinären Grenzen, für Bildung sind alle zuständig. Klären die beteiligten Wissenschaftler aber auch das Thema systematisch, formulieren sie anders oder besser, überbieten sie - um Herbart zu zitieren - das gemeine „Gespräch des Tages“ (Herbart 1813/1982, S. 70), durch das er schon 1813 die Behandlung pädagogischer Fragen belastet fand? 
Zunächst formulieren die beteiligten Wissenschaften, erwartbar schon nach ihrer Zahl, selbst in großer Vielfalt. Bildung wird dann - einerseits - durchaus nahe an der Alltagserwartung definiert oder diskutiert: als „Humankapital“ in der Bildungsökonomie, als „kulturelles Kapital“ in der Bildungssoziologie, für den Zusammenhang von schulischem Lernen, Zertifizierung und Erfolg im Lebenslauf selbst in der Bildungspsychologie oder in der empirischen Bildungsforschung, als gelungene oder missglückte Schulkarriere in der Literatur. Im Nationalen Bildungsbericht - Zentraldokument der empirischen Bildungsforschung und der aktuellen Bildungspolitik - findet sich für Bildung immerhin eine schöne, klare Definition von zentralen Dimensionen des Themas: Bildung bedeute ,individuelle Regulationsfähigkeit, gesellschaftliche Teilhabe und Chancengleichheit sowie Humanressourcen“ (z. B. Autorengruppe Bildungsberichterstattung 2010, S. 1) - und am Ende werden auch hier die „Erträge“ und der ,individuelle Nutzen von Bildung“ diskutiert, abgebildet in Indikatoren zur Qualifikationsstruktur, zu Erwerbschancen und -risiken, zur individuellen Lebensführung und zum Einkommen im Lebenslauf, alles ,,je nach Bildungsstand“ (ebd., S. 193 ff.). Der erzeugt dabei durchaus Differenzen im Blick auf Reichtum, Gesundheit und Lebenschancen, auch wenn man nicht verkennen kann, dass die Qualifikationsstruktur der Arbeitslosen den Hochschulabsolventen genauso kennt wie den Facharbeiter. Selbst die Philosophie arbeitet sich, analytisch, an Bewertungsbegriffen für solche offenbaren Differenzen ab: Bildungsgerechtigkeit und Gleichheit sind hier zentrale Themen, Zugangschancen und Teilhabe zentrale Kriterien.

Harmonie also zwischen den Alltagserwartungen, den politischen Versprechen und der theoretischen Ordnung des Themas? - Es ist wie im alten Gallien: Eine kleine Gruppe von Beobachtern, nicht allein Philosophen, auch nicht nur in der kritischen Pädagogik, sehen Begriff wie Realität der Bildung anders, ja vollständig anders. Sie sehen Bildung gerade nicht dort, wo die Alltagserfahrung und die empirische Bildungsforschung oder die analytische Erziehungsphilosophie sie verorten, in Titel und Stelle, Beruf und Arbeit, Lebenschancen und Zukunftssicherung, sondern an ganz anderer Stelle, theoretisch wie praktisch. Als „Ökonomisierung“" wird diese Perspektive auf Bildung kritisiert (vgl. z. B. Frost 2006), neben Humboldt - der für die Zweckfreiheit stehen muss - werden andere klassische Texte, klassisch auch in der Zuspitzung von Kontroversen bemüht: An den Zusammenhang von Bildung und gesellschaftlicher Brauchbarkeit wird erinnert, den die Philanthropen ungeklärt hinterlassen haben (vgl. Niethammer 1808/1968); der Utilitarismus falscher Aufklärung wird bemüht, sogar, wie bei Evers 1807; Bildungssysteme gelten als Anstalten der „Lebensnot“, wie bei Nietzsche 1872 (Nietzsche 1954, S. 231 f.); im Zertifikat drücke sich allenfalls „Halbbildung“ aus, der klassische Vorwurf seit der Mitte des 19. Jahrhunderts, von Wagenmann (1859, S. 360) über Paulsen (1903/1906, S. 669) bis zu Adorno (1959/1962) vorgetragen, wird erneuert; genauso wie „Verbildung“ oder „Unbildung“ (Liessmann 2006), keine Varianz in der Kritik zwischen 1859 und 2009 also. Phänomene, die sich offenbar immer neu mit Bemühungen zur Qualitätssteigerung im Bildungswesen und allen Versuchen verbinden, die Qualität der Bildung in Beteiligungsraten $\mathrm{zu}$ steigern und $\mathrm{zu}$ messen oder an den kognitiv definierten Ergebnissen schulischer Bildungsarbeit zu prüfen, gelten heute allein als Beleg für den „Selbstwiderspruch der bürgerlichen Gesellschaft“, für „Enteignung des Bewusstseins“ (jüngst erneut: Pongratz 2010). Statt Vernunft, Sittlichkeit, Höherbildung der Menschheit und kritischen 
Vernunftgebrauch zu befördern, also den Kriterien zu genügen, die allein ,wahre Bildung“" ausmachen, wie schon Hegel zu unterscheiden wusste (Hegel 1820/1972, § 187, 2. Zusatz), gibt es dann nur Verfall und Verfehlung des Bildungsanspruchs. Bildung wird, per definitionem, zum Nicht-Messbaren, zum Anderen der alltäglichen Praxis, zur Negation schulisch erwarteter Lernprozesse und -strukturen, zur systematischen Kritik aller empirischen Bildungsforschung. „Wahre Bildung“ kann nicht Ware sein, der Dissens der Rede zeigt einen Widerstreit der Theorie an - einen nicht auflösbaren, wie man hören kann.

Auch diese Argumente haben, im Kreis der Theoretiker und in den Leitartikeln großer Zeitungen, eine gewisse Bekanntheit errungen und ein Schisma befestigt, das den Bildungsdiskurs bis heute charakterisiert. Soll man es dabei belassen? Soll man auf die Rede von Bildung angesichts dieser ungeschlichteten Heterogenität verzichten? Schadet es? - Ich denke es schadet; denn zumindest der theoretische Grundlagendiskurs, aber genauso auch der politische Grundlagenstreit gewinnen nicht an Klarheit und Stringenz, wenn unversöhnliche Positionen undiskutiert regieren oder Disziplinen, wie die Erziehungswissenschaft, ihre Identität auf einem Begriff aufbauen, der keine Klarheit besitzt. Aber kann man überhaupt Lösungen erwarten, gibt es eine Position, über Bildung anders zu reden, jenseits der Ökonomisierungserwartung und -unterstellung, aber auch ,,jenseits von Utopie und Entlarvung“ (Geulen und Pethes 2007), und nicht nur in der Literaturwissenschaft? Ich will es versuchen.

\section{II}

Vielleicht hilft es ja, sich angesichts scheinbar aussichtsloser Konfliktlagen nicht gleich mit Theorien zu beschäftigen, sondern mit dem Thema, das in den Theorien thematisiert wird. Das Thema scheint ja auch, bei allen Kontroversen, nicht strittig: Es geht um das Aufwachsen, und zwar das Aufwachsen in Gesellschaften, unter spezifischen, historisch und gesellschaftlich wie kulturell variablen Bedingungen und Erwartungen. Man könnte diese Struktur des Aufwachsens eine soziale Tatsache nennen. Sie bezeichnet Prozess und Produkt des Aufwachsens und seine Organisation; denn das wird offenbar in allen Kulturen getan, wenn auch in sehr verschiedenen Formen: Schulen z. B. sind weder die Regel noch zwingend, schon gar nicht allgemeine Schulpflicht oder generelle Alphabetisierung, nur: Organisiert wird es, aber immer so, dass die Struktur des Aufwachsens selbst erwartbar wird im Lebenslauf und offenbar auch für die Heranwachsenden unausweichlich.

Dann gilt, z. B. im Blick auf die Erwartungen, dass man ein handlungsfähiges Mitglied der jeweiligen Gemeinschaft wird. Das scheint universell als Erwartung zu gelten; ich kenne jedenfalls keine gegenteilige Strategie oder Zielvorgabe, weder historisch noch ethnologisch. Als ,autonomes Subjekt“ anerkannt zu werden und aufzuwachsen, das klingt dagegen schon sehr westlich. „Individualität“ auszubilden, vielleicht sogar unverwechselbar zu werden, das ist noch jüngeren Datums, zumal dann, wenn man es für alle Individuen gesellschaftlich erwartet.

So vertraut, alltäglich und wiederkehrend diese soziale Tatsache ist, mit der man es $\mathrm{zu}$ tun hat, warum ist die Theoretisierung so problematisch? Zunächst muss man sehen, 
dass die Theoretisierung selbst ein relativ spätes Phänomen ist; jedenfalls dann, wenn man eine theoretisch exklusive - autonome - Theoretisierung sucht, also nicht nur eingebunden in Philosophie oder Theologie (die schon immer wussten, dass Erziehung im Kontext von Staat und Familie wichtig ist) oder in der schönen Literatur oder in der Weisheit einer alltäglichen Lebensführung. Theoriebildung in dem selbstständigen Sinne eines eigenen Wissenssystems ist etwas Neues, historisch relativ Spätes, und es ist ein Indikator für Problemlagen, für das Nicht-Gelingen oder Problematisch-Werden des vorher Selbstverständlichen, des vorher lebensweltlich, traditional und hinreichend geordneten Aufwachsens.

Auf einmal gibt es Alternativen für das Aufwachsen, sogar in durchaus anerkannter Weise: In Mitteleuropa gilt das schon im ausgehenden 18. Jahrhundert, wenn man z. B. dem Theater vertraut und hier Jakob Michael Reinhold Lenz (1751-1792) und seinem „Der Hofmeister“, der in dieser „Komödie“ (1774) die „Vorteile der Privaterziehung“ darstellt. Bei Lenz findet sich ein kleiner Dialog, der über den heranwachsenden Sohn zwischen dem Vater, einem traditionalistisch denkenden Militär, und dessen zukunftsoffenem Bruder, einem Geheimen Rat, geführt wird:

Major: ... Soldat soll er werden; ein Kerl, wie ich gewesen bin. Geh. Rat: Das letzte laß nur weg, lieber Bruder; unsere Kinder sollen und müssen das nicht werden, was wir waren: die Zeiten ändern sich, Sitten Umstände, alles, und wenn du nichts mehr und nichts weniger geworden wärst, als das leibhaftige Konterfei deines Eltervaters (zit. n. Fertig 1979, S. 191).

In diesem Kontext wird „Bildung“ als Medium der sozialen Emanzipation entdeckt; es bezieht sich auf ein Subjekt, dem man Freiheit zuschreibt, und zwar als gesellschaftlich definierte menschliche „Bestimmung“, nicht als göttliche Zuschreibung. Bildung wird dabei als Medium der Selbstkonstruktion des Subjekts gedacht und zwar jenseits der gesellschaftlichen „Einwirkungs“-Strategien, die vielmehr schon kritisch gesehen und als moralisch illegitime Strategien der Unterwerfung des Subjekts bewertet werden. Bildung wird in diesem Kontext auch als Form beschrieben, sich in Gesellschaft zu bewegen, als „Politur“ des Geistes (Mendelssohn 1784); jedenfalls wird der Heranwachsende nicht länger so toleriert, wie sich z. B. der junge Lessing sah (und seiner Mutter zur Rechtfertigung seiner Bildungsaktivitäten am 20.1.1749 aus Berlin mitteilt): „Eine bäuersche Schichternheit, ein verwilderter und ungebauter Körper, eine gäntzliche Unwissenheit in Sitten und Umgange“, dem er sofort abhelfen will:.,,Wenn ich auf meiner Wanderschafft nichts lerne, so lerne ich mich doch in die Welt schicken." (Lessing 1749, zit. Fertig 1991, S. 22, S. 25)

„Bildung“ bedeutet deshalb auch den „Umgang mit Menschen“, seine „Zivilisierung“ und „Kultivierung“, wie von Knigge bis Kant gesagt wird. Bildung stellt also einen Begriff dar, in dem sich Gesellschaft in ihrer Zeitlichkeit und offenen Zukunft selbst thematisiert und eine soziale Form definiert, in der sich die individuelle Form des Aufwachsens mit den gesellschaftlich gewünschten Zuständen verbinden lässt, als Erwartbarkeit unseres Verhaltens für andere - als Bildung eben. Deshalb kommt dem Begriff auch zeitdiagnostische Valenz zu, weil mit der Entstehung und Verwendung dieser Form der Selbstbeschreibung zugleich die Frage aufkommt, ob das, was den Subjekten im Auf- 
wachsen geschieht, legitim ist und ob die Wechselwirkung von Mensch und Welt in einer Welt geschieht, die solcher Wechselwirkung förderlich ist. Deshalb lesen sich bereits die frühen Bildungstexte wie kritische Zeitdiagnosen und von Entfremdung als Struktur des Mensch-Welt-Verhältnisses, von der Schiller in den „Briefen zur ästhetischen Erziehung“ (Schiller 1794/1795/1966) spricht und die Humboldt schon als „Entfremdung“ bezeichnet (Humboldt 1793/1980b, S. 237).

Ein weiterer Anlass, Protoformen der Theorie, also neues Wissen über das Aufwachsen auszubilden, besteht wegen der Erfahrung von Differenz in den Ergebnissen des Aufwachsens, vor allem wegen der Differenz von Erwartung und Ergebnis der privaten wie öffentlichen Erziehung. Höherbildung ist nicht das alltägliche Ergebnis, obwohl „Moralisierung" erwartet wird, wenn Bildung thematisch ist. Andere, neue Beobachter der Erziehung, z. B. ein Schweizer Pädagoge und pädagogischer Unternehmer, versprechen dann sogar das Verschwinden der Kriminalität, wenn man Kinder nur einer ordentlichen öffentlichen Erziehung zuführt. „Bildung“ wird insofern auch auf das ganze Gemeinwesen ausgelegt, damit sogar zum Medium, die „Nation“ zu erzeugen, also die Gemeinschaft derjenigen, die verständig und nach universalistischen Kriterien die öffentlich bedeutsamen Fragen regeln.

Gleichzeitig stellt man bei der Lektüre der Quellen fest: Explizite Texte mit dem Titel „Theorie der Bildung“ erscheinen in dieser Zeit noch nicht. Ein heute in der Theorie der Bildung berühmtes Textfragment - meist als „Theorie der Bildung“ (Humboldt 1793/1980b) publiziert - hat diesen Namen erst 100 Jahre später in der Zuschreibung durch einen Editor erhalten. Andere Formen der Reflexion des Aufwachsens, die durchaus das Thema der Bildung aufnehmen, haben auch nicht Theorie- oder Philosophiestatus, sondern den Status des Romans, z. B. eines ,psychologischen Romans“, wie beim „Anton Reiser“ (1785) des Karl Philipp Moritz, oder schlicht nur eines „Romans“, wie bei Goethes „Wilhelm Meisters Lehrjahre“ (1795/96), der heute als das klassische Exempel eines „Bildungsromans“ gilt und ja auch Bildung im neuen Verstande thematisiert: „mich selbst, ganz wie ich da bin, auszubilden, das war dunkel von Jugend auf mein Wunsch" schreibt Wilhelm (in seinem Brief an Werner, V, 3). Das ist zwar Thematisierung von Bildung, auch unter dem modernen Anspruch der Selbstkonstruktion des Subjekts, aber doch nicht Theorie.

Wo gibt es diese Bildungstheorie als Theorie des Aufwachsens von Subjekten? Trotz aller Bemühungen meiner philosophischen Freunde, eine solche Theorie des Aufwachsens kann ich nicht in Hegels „Phänomenologie des Geistes“ erkennen, obwohl dort unablässig von „Bildung“ die Rede ist; aber damit ist doch kaum der Bildungsprozess empirischer Subjekte gemeint (dafür sollte man vielleicht eher die Rechtsphilosophie lesen). Auch in den kantischen Kritiken sehe ich, zahlreicher pädagogischer Exegesen ungeachtet, keine Theorie des Aufwachsens; ,das transzendentale Subjekt“ ist nicht im Kindergarten zu finden oder in der Schule (auch wenn das in Wien Verstimmung auslöst), die „Kritik der Urteilskraft“ liefert keine Entwicklungspsychologie oder Anthropologie und die „Kritik der praktischen Vernunft“ konkurriert nicht mit Piagets Empirie der Entstehung des moralischen Urteils.

Das Aufwachsen selbst, um zu den Theorien zu kommen, wird woanders behandelt, z. B. in der „Erfahrungsseelenkunde“, und hier schon bereits in strikter Abgrezung gegen 
manche Varianten der Philosophie, denn das Motto ist „Fakta, und kein moralisches Geschwätz“ (Moritz 1783, S. 2). Das Thema wird schon historisch als theoretisches Thema aber vor allem in der „Pädagogik“ behandelt, auch bei Kant, und es gehört in den Kontext der empirischen und pragmatischen Anthropologie und es geht um ,physische“ und „moralische Erziehung“. Schleiermachers Grundbegriff in den Pädagogik-Vorlesungen ist ebenfalls der der „Erziehung“, Herbart schreibt eine „Allgemeine Pädagogik“ und denkt über einen „Unterricht“ nach, „der erzieht“. Bildsamkeit, eine anthropologischnaturphilosophische Prämisse über die Möglichkeit der Selbstkonstruktion des Menschen, ist sein „Grundbegriff der Pädagogik“; auch Fröbel spricht nur von der „Menschenerziehung“ und gründet unablässig „Erziehungsanstalten“, in denen immer und immer wieder erzogen wird.

Erziehung, nicht Bildung wird m. a. W. in der sich ausdifferenzierenden Reflexion über das Aufwachsen - also jenseits der philosophischen Texte - zunächst zum theoretisch leitenden Begriff, vor allem dann, wenn diese soziale Tatsache in eigener theoretischer und methodischer Anstrengung als Grundlage einer eigenständigen Wissenschaft beobachtet werden soll. Als am Ende des Vormärz die Pädagogen anfangen, ihr eigenes Geschäft und ihre disziplinäre Grundbegrifflichkeit mit dem Begriff der Bildung zu belegen (durch die höheren Schulen verführt, in denen klassische „Bildung“ das Thema ist und „Bildung“ auf den Umgang mit den richtigen „Bildungsgütern“ verkürzt wird), warnt sie der Hegelianer Karl Rosenkranz ausdrücklich vor dem drohenden Irrweg: Der Grundbegriff der Pädagogik als Wissenschaft sei der Begriff der „Erziehung“; der Begriff der Bildung dagegen gehöre allen Humanwissenschaften, als verbindende Kategorie, vor aller Spezifikation, insofern nur philosophisch präsent (Rosenkranz 1848, S. VIII, S. 9 ff.). Dort findet man ihn dann ja auch im 19. Jahrhundert, von der Rechtsphilosophie und der praktischen Philosophie bis zu den Verwaltungswissenschaften und der Nationalökonomie.

Es ist im Übrigen niemand anders als Wilhelm Dilthey, der in seinem ,System der Pädagogik“ die Warnung von Rosenkranz vor dem Begriff der Bildung als konstitutivem Begriff disziplinär organisierter Reflexion wiederholt, die Rückkehr zur „Erziehung“ empfiehlt und zu der Grundtatsache aller pädagogischen Theorie, und die heißt für Dilthey lapidar: „Die Erziehung ist eine Funktion der Gesellschaft“ (Dilthey 1884/1894, S. 192).

Vor diesem Hintergrund ist es eine durchaus überraschende Pointe der Wissenschaftsgeschichte, dass ausgerechnet Diltheys Erben sich der „Bildung“ in die Arme werfen, um die wissenschaftliche - die ,philosophische“ - Pädagogik als Disziplin zu begründen (u. a. Spranger 1933/1934), die Theoretisierung der Erziehung in einer eigenen „Erziehungswissenschaft“ dagegen strikt abwehren (ja die Erziehungswissenschaft, wie Wilhelm Flitner einmal sagte, für eine Erfindung aus den Statusaspirationen der LehrerGewerkschaften halten), und die Erziehung als Praxis den pädagogischen Reformbewegungen und der Selbsterziehung in Jugendkulturen überlassen. Es ist schließlich ein noch schlechteres Datum der Begriffsgeschichte, dass der Begriff der Erziehung bald sogar vollständig negativ konnotiert wird. Bei Heinz-Joachim Heydorn erreicht diese wertthematische Disjunktion von Bildung und Erziehung ihren Höhepunkt. „Erziehung“, das ist für ihn „Einfügung, Unterwerfung, Herrschaft des Menschen über den Menschen..., bewusstloses Erleiden“, sogar der „Weg durch das Zuchthaus der Geschichte“ (Heydorn 
1970/1979, S. 9); Bildung dagegen die „Antithese“ zur Erziehung, „entbundene Selbsttätigkeit ... schon vollzogene Emanzipation ... neue, geistige Geburt ... Versöhnung des Menschen mit sich selbst" (ebd., S. 10 f.).

Am Ende steht der (einer verkürzten Emile-Lektüre scheinbar begründet abgewonnene) rousseauistische Widerspruch von Mensch und Bürger, radikalisiert sogar, aber das kantische „Problem“ der Pädagogik, der Anfang aller eigenständigen theoretischen Thematisierung - ,Wie kultiviere ich die Freiheit bei dem Zwange?“ (Kant 1803/1964, A 32, S. 711) - wird dem gesellschaftskritischen Verdikt geopfert. Herbarts nüchtern-lapidare Kritik an allen solchen Kritik-Theorien: „Der Mensch ist Nichts ausser der Gesellschaft ..." (Herbart 1824/1925, Bd. 2, S. 3) wird ignoriert. Die für die Moderne charakteristische, ja notwendige, paradoxe Gleichzeitigkeit der Prozesse der Vergesellschaftung und der Individualisierung, die um 1800 als Thema präsent war, wird nicht mehr gesehen, schon gar nicht theoretisch bearbeitet, sondern zum unauflöslichen Widerspruch stilisiert. Bildung reduziert sich auf Zeitdiagnostik und Kritik, erst auf Kulturkritik und „Kulturpädagogik“, dann auf „kritische Zeitdiagnostik“. Erziehungswissenschaft gilt vielen nur als „kritische“ als legitim, vielleicht sogar nur als Duplikation der Kritischen Theorie.

\section{III}

Unsere aktuelle Situation der „Bildung“ als Reflexionsform hat also diese historisch aufklärbare Struktur. Unsere Begriffe für das Aufwachsen sind nicht zufällig vielfach präsent; sie entsprechen der Historizität der Thematisierung des Themas und der Gleichzeitigkeit von Selbstbeschreibungen und distanzierten Beobachtungen. „Bildung“, als Selbstbeschreibung moderner Gesellschaften, und „Erziehung“, als theoretischer Begriff für die Struktur der sozialen Tatsache, stehen in der Erziehungswissenschaft relativ unverbunden nebeneinander. In anderen theoretischen Kontexten der Beobachtung des Aufwachsens treten weitere Begriffe hinzu: „Sozialisation“ z. B. oder „Lernen“, auch „Identitätskonstruktion“ oder „Identitätsbildung“, wenn man auf das im Prozess erwartete Produkt verweisen will. Jede Disziplin sucht ihre Spezifik in der Theoretisierung des Aufwachsens in Gesellschaften. Rosenkranz wie Dilthey können sich darin bestätigt sehen, dass das Thema Bildung nicht disziplinär exklusiv zurechenbar ist oder auch nur, dass sich die Thematisierungsweisen gegeneinander aufrechnen lassen. Historisch wird man auch die Kontinuität der Zeitdiagnostik gut verstehen - sie wird beliebt in dem Maße, in dem Optionen zukünftiger Entwicklung von Mensch und Welt nicht nur in größerer Zahl existieren, sondern auch mehr Akteuren tatsächlich offen stehen und sich zugleich Exempel für die Differenz von universalen Versprechen und individueller oder kollektiver Realisierungschance unabweisbar aufdrängen.

Ist deshalb alles in Ordnung, leben wir ruhig weiter in der Konkurrenz der Thematisierungsweisen, lösen wir sie als Ko-Präsenz auf, die nur in Verkennung der kategorialen Grundstruktur sich als wechselseitige Kritik interpretieren lässt? Das mag Frieden stiften und von Legitimationszwängen entlasten, wenn man weiß: Die Empiriker wie die kritischen Kritiker reden weder über dasselbe Phänomen, weil sie den theoretischen Gegenstand nicht teilen, noch reden sie in derselben Form der Thematisierung. Die Rede 
von Bildung erweist sich im besten Fall als ein überwölbendes Dach, als Begriff, der Relationen zwischen Disziplinen stiften kann, ist man bereit zuzugestehen, dass Bildung sich - in allen Varianten der realitätszugewandten Bildungsforschung - als grundlegende (wenn auch nicht konkurrenzlose), aber inzwischen als theoretisch fruchtbar ausgewiesene These über den Prozess des Aufwachsens lesen lässt: Im Begriff der Bildung ist dann die These von der Selbstkonstruktion des Subjekts artikuliert, allerdings in den paradoxen Bahnen Kants, die der Kultivierung der Freiheit bei dem Zwange gelten, oder in den Erläuterungen des Marx vom 18. Brumaire: „Die Menschen machen ihre eigene Geschichte, aber sie machen sie nicht aus freien Stücken ..." (Marx 1852/1971, S. 226).

Der Rest, jenseits der sozialphilosophischen Basisprämisse, ist Forschung. Auch für die Philosophie der Erziehung bleibt dann neben der Fixierung des Themas vielleicht noch die zeitdiagnostische Arbeit, falls es die jenseits empirischer Forschung noch legitim gibt. Berücksichtigt man nämlich die unverkennbare Tatsache, dass heute zumindest eine bestimmte „Variante“ kritischer Zeitdiagnose, die auch im disziplinären Diskurs der Pädagogik sehr beliebt war und ist, ganz offenkundig den „Augenblick ihres Entschwindens“ erlebt, dann ist auch dieses Thema verschwunden, d. h. „eine Kritik, die sich als Inhaberin des überlegenen Standpunktes wähnte - eines Standpunktes, der sich klassischerweise auf Mastersubjekte wie die Wahrheit, die Vernunft und die Geschichte berief. Mit dieser Art der Kritik und dem Gestus der starken Behauptung, der Einschüchterung und der Unterwerfung, ist es nun vorbei.“ (Konersmann 2008, S. 7)

Soll, kann man sich deshalb auf solche irenische Positionen zurückziehen, kategoriale Differenzen klären und die Möglichkeiten der grundlagentheoretischen Kooperation wie die Schwächen der kritischen Tradition sehen? Ich würde das nicht empfehlen, denn zwei Erwartungen an eine Theorie des Aufwachsens in Gesellschaften werden mit der puren Tatsache der Vielfalt und des Nebeneinanders differenter, aber nicht konkurrierender Theorien überhaupt nicht gelöst: Kants Problem einerseits und die Frage nach der Legitimität der öffentlichen, zumal schulischen Erziehung andererseits.

Mit Kant muss man fragen: „Wie ist Erziehung möglich?“, also revierspezifisch die Basisfrage an alle modernen Erfahrungswissenschaften aufwerfen, wie möglich ist, was wirklich ist: Recht, Ökonomie, Wissenschaft (etc.) - und Erziehung. Die Antworten auf diese Wie-Frage gibt es nur empirisch, nicht philosophisch; denn schon bei Kant ist klar, „aus Prinzipien“ kann man nicht ableiten, wie solche Muster der Entparadoxierung möglich werden, die sich bei der Aufgabe stellen, „die Freiheit bei dem Zwange zu kultivieren". Die Tradition einer bildungstheoretischen Frage verweist also notwendig und nicht zufällig auf empirische, d. h. auf realitätszugewandte und -kontrollierte Bildungsforschung, sogar mit eindeutigen Konsequenzen:

- Dann ist z. B. die aktuell dominierende outcome-orientierte Messung nicht bildungstheoretisch verboten, sondern durchaus legitim, aber für die Prozessprobleme unzureichend, ja sogar theoretisch (allmählich) uninteressant;

- dann ist Schule nur im Kontext des Lebenslaufs angemessen in ihrer spezifischen pädagogischen Leistung und Wirkung interpretierbar, als Raum der Ermöglichung in einem Spiel von Zurechnung und Selbstkonstruktion - und zwar in Varianz, nicht als System der Garantie schöner Welten und guter Menschen; 
- dann muss man sich von einer deterministischen Theorie verabschieden, die den Menschen als Objekt der Institution sieht, und sich einer der sozialen Tatsache des Aufwachsens angemessenen Theorie zuwenden, die „Bildung“ als die genuine Form versteht, in der sich Funktion und Intention, Alltäglichkeit der Subjekte und die Omnipräsenz der Sozialsysteme verschränken;

- Kritik speist sich deshalb auch nicht aus der Gleichzeitigkeit von Individuation und Vergesellschaftung (das wäre schlechte Negation der Wirklichkeit), sondern aus der Frage, wie man diese Schnittstelle besser oder schlechter gestalten kann; denn „richtig“ ist als Kriterium bei Situationen der Entparadoxierung unangebracht. Man muss immer mit Ameliorierung und Demeliorierung rechnen, weil man es mit selbstbestimmten Subjekten zu tun hat, die auch die Urteilskraft reflektierender Subjekte besitzen, also auch das schönste pädagogische Programm und selbst die Einsichten kritischer Kritiker negieren können;

- Kritik lebt deshalb am ehesten auch als Verweis auf andere Möglichkeiten, beobachtend und anbietend; das eröffnet und verlangt aber nur weitere Empirisierung, nicht kritische Zeitdiagnose.

Auf Kants Frage muss man deshalb antworten: Erst in der empirischen Bildungsforschung in der disziplinären Vielfalt, aus der sie besteht, kommt die theoretisch geleitete Analyse der Möglichkeit und Wirklichkeit von Bildung zu ihrem Recht (und nur am Rande muss man gegenüber ängstlichen oder philosophisch und moralisch fixierten Bildungstheoretikern ergänzen, dass das Attribut „empirisch“ eine Vielfalt von methodischen Möglichkeiten des Realitätszugangs meint).

„Ist Erziehung sittlich erlaubt?“, das ist die andere Frage; sie stellt sich nicht allein für die Moderne oder nur kapitalismuskritisch, sondern universell. Sie klingt angesichts der Allgegenwart der Erziehung und des Fortschreitens von Pädagogisierung vielleicht etwas artifiziell und hilflos oder in der Erziehungskritik als schon beantwortet, aber die Erfahrung von Bedrohung und Missbrauch in Erziehungseinrichtungen erinnert daran, dass die Frage nicht überflüssig ist. Hier hätte Erziehungsphilosophie ihr auch moralisch relevantes Feld der Reflexion. Jenseits des Strafrechts, das nur nachgehend klärt und zurechnet, könnte man sich ja eine pädagogische Ethik vorstellen, die im Prozess selbst das Handeln des Pädagogen regiert, unter der bildungstheoretischen Prämisse, dass wir als Pädagogen in Prozesse eintreten, die nicht ein mündiges Subjekt erzeugen, sondern ein zur Selbstorganisation fähiges Subjekt schon voraussetzen. Wir „,kultivieren die Freiheit bei dem Zwange", wir erzeugen sie pädagogisch nicht.

Im Bildungsroman war die dann wartende Schwierigkeit im Übrigen durchaus präsent, wenn der Wilhelm Meister der Lehrjahre im Umgang mit Felix nämlich erkennt, „dass wirklich der Knabe mehr ihn, als er den Knaben erzieht“. Advokatorisch kann die pädagogische Ethik dann allein nicht mehr sein; sie basiert eher auf Anerkennung, d. h. auf die im strukturell asymmetrischen Prozess des Erziehens immer auch unterstellte Symmetrie - und sie müsste so stark sein, das sie auch präventiv funktioniert, ohne den Strafrichter. Das zu entwickeln und im professionellen Habitus zu realisieren, das wäre ein reformpädagogisches Projekt, wunderbar zu wünschen - und wenn uns die Tradition des Bildungsdenkens dazu führt, warum denn nicht? Angst haben wir nur vor dem bösen Wolf, nicht vor der Tradition der Bildung. 
Open Access Dieser Artikel unterliegt den Bedingungen der Creative Commons Attribution Noncommercial License. Dadurch sind die nichtkommerzielle Nutzung, Verteilung und Reproduktion erlaubt, sofern der/die Originalautor/en und die Quelle angegeben sind.

\section{Literatur}

Adorno, T. W. (1962). Theorie der Halbbildung [1959]. In T. W. Adorno \& M. Horkheimer (Hrsg.), Sociologica II (S. 168-192). Frankfurt a. M.: Suhrkamp.

Autorengruppe Bildungsberichterstattung. (2010). Bildung in Deutschland 2010. Ein indikatorengestützter Bericht mit einer Analyse zu Perspektiven des Bildungswesens im demografischen Wandel. Im Auftrag der Ständigen Konferenz der Kultusminister der Länder in der Bundesrepublik Deutschland und des Bundesministeriums für Bildung und Forschung. Bielefeld: W. Bertelsmann.

Dilthey, W. (1894). Pädagogik. Geschichte und Grundlinien des Systems [1884] (Gesammelte Schriften, IX. Bd, 4. Aufl.). Göttingen: Vandenhoeck \& Ruprecht.

Evers, E. A. (1807). Über die Schulbildung zur Bestialität. Aarau: Friedrich Jacob.

Fertig, L. (1979). Die Hofmeister. Ein Beitrag zur Geschichte des Lehrerstandes und der bürgerlichen Intelligenz. Stuttgart: J. B. Metzler.

Fertig, L. (Hrsg.). (1991). Bildungsgang und Lebensplan. Briefe über Erziehung von 1750 bis 1900. Darmstadt: Wissenschaftliche Buchgesellschaft.

Frost, U. (Hrsg.). (2006). Unternehmen Bildung. Die Frankfurter Einsprüche und kontroverse Positionen zur aktuellen Bildungsreform (Sonderheft zur Vierteljahrsschrift für wissenschaftliche Pädagogik). Paderborn: Schöningh.

Geulen. E., \& Pethes, N. (Hrsg.). (2007). Jenseits von Utopie und Entlarvung. Kulturwissenschaftliche Untersuchungen zum Erziehungsdiskurs der Moderne. Freiburg i. Br.: Rombach.

Hegel, G. W. F. (1972). Grundlinien der Philosophie des Rechts oder Naturrecht und Staatswissenschaft im Grundrisse [1820]. Hrsg. und eingeleitet von H. Reichelt. Frankfurt a. M.: Ullstein.

Herbart, J. F. (1824/1825). Psychologie als Wissenschaft, neu gegründet auf Erfahrung, Metaphysik und Mathematik (Bd. 2). Königsberg: Unzer.

Herbart, J. F. (1982). Über pädagogische Diskussionen und die Bedingungen unter denen sie nützen können [1813]. In W. Asmus (Hrsg.), Herbart. Pädagogische Schriften (Bd. 3, S. 69-72). Stuttgart: Klett.

Heydorn, H.-J. (1979). Über den Widerspruch von Bildung und Herrschaft [1970] (Bildungstheoretische Schriften 2). Frankfurt a. M.: Europäische Verlagsanstalt.

Humboldt, W. v. (1980a). Ideen zu einem Versuch, die Gränzen der Wirksamkeit des Staates zu bestimmen [1792]. In A. Flitner \& K. Giel (Hrsg.), Humboldt. Werke in fünf Bänden (Bd. I, 3. Aufl., S. 56-233). Darmstadt: Wissenschaftliche Buchgesellschaft.

Humboldt, W. v. (1980b). Theorie der Bildung des Menschen [1793]. In A. Flitner \& K. Giel (Hrsg.), Humboldt. Werke in fünf Bänden (Bd. I, 3. Aufl., S. 234-240). Darmstadt: Wissenschaftliche Buchgesellschaft.

Kant, I. (1964). Über Pädagogik [1803]. In W. Weischedel (Hrsg.), Kant. Werke in zehn Bänden (Bd. 10, S. 691-761). Darmstadt: Wissenschaftliche Buchgesellschaft.

Konersmann, R. (2008). Kulturkritik. Frankfurt a. M.: Suhrkamp.

Liessmann, K. P. (2006). Theorie der Unbildung. Die Irrtümer der Wissensgesellschaft. Wien: Zsolnay.

Marx, K. (1971). Der achtzehnte Brumaire des Luis Bonaparte [1852]. In Karl Marx - Friedrich Engels. Ausgewählte Schriften in zwei Bänden (Bd. 1, S. 226-316). Berlin: Dietz.

Mendelssohn, M. (1784). Über die Frage: Was heißt Aufklären? Berlinische Monatsschrift, (2)9. Stück, 193-200. 
Moritz, C. P. (Hrsg.). (1783). ГNQ $\theta I$ IAYTON [Gnōthi sauton] oder Magazin zur Erfahrungsseelenkunde. Berlin: August Mylius.

Niethammer, F. I. (1968). Der Streit des Philanthropinismus und Humanismus in der Theorie des Erziehungs-Unterrichts unsrer Zeit [1808] (Nachdruck, Faksimile). In W. Hillebrecht (Hrsg.), Friedrich Immanuel Niethammer: Philanthropinismus - Humanismus. Texte zur Schulreform (S. 81-445). Weinheim: Beltz.

Nietzsche, F. (1954). Über die Zukunft unserer Bildungs-Anstalten. Vierter Vortrag [5. März 1872]. In K. Schlechta (Hrsg.), Friedrich Nietzsche. Werke in drei Bänden (Bd. 3, S. 229-247). München: Hanser.

Paulsen, F. (1906). Bildung [1903]. In W. Rein (Hrsg.), Enzyklopädisches Handbuch der Pädagogik (2. Aufl., Bd. 2, S. 658-670). Langensalza: Beyer \& Mann.

Pongratz, L. (2010). Sackgassen der Bildung. Pädagogik anders denken. Paderborn: Schöningh.

Rosenkranz, K. (1848). Die Pädagogik als System. Königsberg: Gebrüder Bornträger.

Schiller, F. (1966). Über die ästhetische Erziehung des Menschen in einer Reihe von Briefen [1794/1795]. In H. G. Göpfert (Hrsg.), Schiller. Werke in drei Bänden (Bd. 2, S. 445-520). München: Hanser.

Spranger, E. (1933/1934). Umrisse der philosophischen Pädagogik. Internationale Zeitschrift für Erziehungswissenschaft, 3, 160-180, 332-337, 448-467 [auch in: Philosophische Pädagogik (Gesammelte Schriften, Bd. 2, S. 7-61). Heidelberg: Quelle \& Meyer, 1973].

Wagenmann, A. (1859). Ausbildung. In K. A. Schmid (Hrsg.), Encyklopädie des gesammten Erziehungs- und Unterrichtswesens (Bd. 1, S. 357-361). Gotha: Rudolf Besser. 